Mathematical Research Letters 11, 197-211 (2004)

\title{
A CONVEXITY PROPERTY FOR REAL ORBITS IN COMPLEXIFIED RIEMANNIAN SYMMETRIC SPACES
}

\author{
BERNHARD KRÖTZ
}

\section{Introduction}

For real semisimple Lie groups $G$ and their Lie algebras $\mathfrak{g}$ interesting features of convexity have been discovered. This paper makes a contribution which is related to certain $G$-orbits in the complexification $X_{\mathbb{C}}=G_{\mathbb{C}} / K_{\mathbb{C}}$ of a Riemannian symmetric space $X=G / K$ of the non-compact type. Applications to analytically continued spherical functions will be given.

Our results borrow in spirit and techniques from the well understood situation on the real space $X$. Therefore it is useful to review some of these results and methods. Let $\mathfrak{g}$ and $\mathfrak{k}$ denote the Lie algebras of $G$ and $K$. Then there is a Cartan decomposition $\mathfrak{g}=\mathfrak{k}+\mathfrak{p}$. We fix a maximal abelian subspace $\mathfrak{a} \subseteq \mathfrak{p}$. Then Kostant's linear convexity theorem [5] reads

$$
(\forall Y \in \mathfrak{a}) \quad \operatorname{pr}(\operatorname{Ad}(K) Y)=\operatorname{conv}(\mathcal{W} Y)
$$

In this formula pr: $\mathfrak{p} \rightarrow \mathfrak{a}$ is the orthogonal projection, $\mathcal{W}=Z_{K}(\mathfrak{a}) \backslash N_{K}(\mathfrak{a})$ is the Weyl group and $\operatorname{conv}(\cdot)$ denotes the convex hull of $(\cdot)$.

There is a related version of (1) on the Riemannian symmetric space $X=$ $G / K$. For that let $G=N A K$ be an Iwasawa decomposition of $G$ and $a: G \rightarrow$ $A$ the corresponding middle projection. Then Kostant's non-linear convexity theorem [5] says

$$
(\forall Y \in \mathfrak{a}) \quad \log a(K \exp (Y))=\operatorname{conv}(\mathcal{W} Y)
$$

Let us mention that for all applications of (1) and (2) to harmonic analysis one only needs the inclusion " $\subseteq$ ". For this part (1) and (2) can easily seen to be equivalent: Let $\lambda \in \mathfrak{a}^{*}$ and $Y \in \mathfrak{a}$ be both regular. Then with $M=Z_{K}(\mathfrak{a})$ the linear/non-linear phase functions on $M \backslash K$ are defined by

$$
\begin{gathered}
f_{\lambda, Y}: M \backslash K \rightarrow \mathbb{R}, \quad M k \mapsto \lambda(\operatorname{pr}(\operatorname{Ad}(k) Y)) \\
F_{\lambda, Y}: M \backslash K \rightarrow \mathbb{R}, \quad M k \mapsto a(k \exp (Y))^{\lambda} .
\end{gathered}
$$

Received May 9, 2003

The work was supported in part by NSF-grant DMS-0097314 
Standard arguments show that $f_{\lambda, Y}$ and $F_{\lambda, Y}$ have the same critical set which is computed to be $\mathcal{W}$ (cf. [12]). From that one easily derives both inclusions " $\subseteq$ " in (1) and (2).

The phase function $F_{\lambda, Y}$ (as well as $f_{\lambda, Y}$ ) is Morse. This means that the method of stationary phase applies for computing the asymptotics of the oscillatory integral associated to $F_{\lambda, Y}$ (cf. [12]). Up to a spectral shift by $\rho \in \mathfrak{a}^{*}$ this oscillatory integral is the spherical function $\varphi_{\lambda}$ on $X=G / K$

$$
\varphi_{\lambda}(a)=\int_{M \backslash K} F_{\rho-i \lambda, Y}(M k) d M k \quad(a=\exp (Y)) .
$$

The conclusion is that convexity associated to the Riemannian symmetric space $X=G / K$ is intimately related to the asymptotics of spherical functions in the spectral parameter $\lambda$. It is important for us that this dictionary remains true once we analytically continue from $X$ to certain domains in $X_{\mathbb{C}}$ (although the method of stationary phase does not apply anymore). We will comment more on this later.

There is another convexity result for $X$ which is related to the polar decomposition $X=K \overline{A^{+}} K / K$ :

$$
\left(\forall Y_{1}, Y_{2} \in \overline{\mathfrak{a}^{+}}\right) \quad \exp \left(Y_{1}\right) K \exp \left(Y_{2}\right) \subseteq K \exp \left(\operatorname{conv}\left(\mathcal{W}\left(Y_{1}+Y_{2}\right)\right)\right) K
$$

(cf. [11])

The main result of this paper is a certain complex version of (3). Let us be more specific. Denote by $\Sigma$ the root system of $\mathfrak{g}$ w.r.t. $\mathfrak{a}$ and define a bounded convex domain in $\mathfrak{a}$ by

$$
\Omega=\left\{Y \in \mathfrak{a}:(\forall \alpha \in \Sigma)|\alpha(Y)|<\frac{\pi}{2}\right\}
$$

If $x_{o}$ denotes the base point in $X_{\mathbb{C}}$, then we shall be interested in those $G$-orbits in $X_{\mathbb{C}}$ which pass through $\exp (i \Omega) \cdot x_{o}$. The union of those orbits gives us the complex crown of the Riemannian symmetric space $X$ :

$$
\Xi=G \exp (i \Omega) \cdot x_{o} \subseteq X_{\mathbb{C}}
$$

The complex crown is a $G$-invariant Stein neighborhood of $X$ in $X_{\mathbb{C}}$ which was subject of many recent investigations.

Finally we set $X_{\mathbb{C}, s}=K_{\mathbb{C}} A_{\mathbb{C}} \cdot x_{o}$ and notice that $X_{\mathbb{C}, s}$ is set of all points in $X_{\mathbb{C}}$

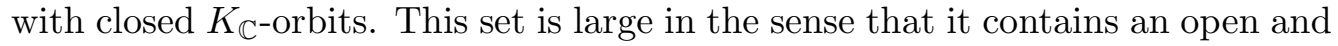
dense subset of $X_{\mathbb{C}}$ (regular semisimple elements). The appropriate holomorphic extension of (3) now is (cf. Theorem 2.1 and Corollary 2.2 below):

Theorem 1. Let $Y \in \Omega$. Then

$$
G \exp (i Y) \cdot x_{o} \bigcap X_{\mathbb{C}, s} \subseteq K_{\mathbb{C}} A \exp (i \operatorname{conv}(\mathcal{W} Y)) \cdot x_{o}
$$


In particular, $\Xi \bigcap X_{\mathbb{C}, s} \subseteq K_{\mathbb{C}} A \exp (i \Omega) \cdot x_{o}$.

For the Lorentzian groups $G=\mathrm{SO}_{e}(1, n)$ this convexity inclusion was proved in [7] by explicit computation. In the classical cases of $G=\operatorname{Sl}(n, \mathbb{K})$, i.e. when $X$ has a canonical realization in symmetric matrices, it might be interesting to observe that Theorem 1 is not obvious from elementary linear algebra (see Section 3 for a detailed discussion).

We describe briefly the method of the proof. It was observed in [8] that spherical functions $\varphi_{\lambda}$ on $X$ admit a holomorphic continuation $\widetilde{\varphi}_{\lambda}$ to $\Xi$. Equally spherical functions extend to $K_{\mathbb{C}}$-invariant holomorphic functions on $K_{\mathbb{C}} A \exp (2 i \Omega) \cdot x_{o}$ (cf. [8]). For fixed generic elements $a \in A \exp (i \Omega)$ we give near optimal estimates for $\widetilde{\varphi}_{\lambda}(a)$ for large spectral parameters $\lambda$. The upper estimate hereby is obtained from the complex version of the non-linear convexity theorem [2]. For the lower estimate we use Harish-Chandra's power series expansion for spherical functions. From these estimates one then derives the convexity inclusion of Theorem 1 .

The above convexity theorem features various applications to harmonic analysis. In summary we show (cf. Theorem 2.3.1 and Theorem 2.3.2 below):

Theorem 2. The following assertions hold:

(1) Let $\varphi_{\lambda}$ be a positive definite spherical function on $X$. Then its holomorphic extension $\widetilde{\varphi}_{\lambda}$ to $\Xi$ is bounded.

(2) Let $k_{t}$ be the heat kernel on $X$ and $\widetilde{k}_{t}$ its holomorphic continuation to $\Xi$ (cf. [9]). Then $\widetilde{k}_{t}$ is bounded.

\section{Basic facts on $K_{\mathbb{C}}$-double cosets and complex crowns}

This section is organized as follows. We begin with introducing the standard notation neded for the discussion of Riemannian symmetric spaces $X=G / K$ and their complexifications $X_{\mathbb{C}}=G_{\mathbb{C}} / K_{\mathbb{C}}$. Then we summarize the basic facts of the $K_{\mathbb{C}}$-action on the affine variety $X_{\mathbb{C}}$. Finally we provide the reader with the definition and main features of the complex crown $\Xi \subseteq X_{\mathbb{C}}$ of a Riemannian symmetric space $X$.

1.1. Notation. Let us denote by $\mathfrak{g}$ a real semisimple Lie algebra with Cartan decomposition $\mathfrak{g}=\mathfrak{k}+\mathfrak{p}$. We fix a maximal abelian subspace $\mathfrak{a} \subseteq \mathfrak{p}$ and set $\mathfrak{m}=\mathfrak{z} \mathfrak{k}(\mathfrak{a})$. Then one has the root space decomposition

$$
\mathfrak{g}=\mathfrak{a} \oplus \mathfrak{m} \oplus \bigoplus_{\alpha \in \Sigma} \mathfrak{g}^{\alpha}
$$

with $\Sigma \subseteq \mathfrak{a}^{*}$ the corresponding restricted root system. Select a positive system $\Sigma^{+} \subseteq \Sigma$ and form the nilpotent subalgebra $\mathfrak{n}=\bigoplus_{\alpha \in \Sigma^{+}} \mathfrak{g}^{\alpha}$.

If $\mathfrak{l}$ is a Lie algebra, then we write $\mathfrak{l}_{\mathbb{C}}$ for its complexification.

On the group level we let $G_{\mathbb{C}}$ be a simply connected Lie group with Lie algebra $\mathfrak{g}_{\mathbb{C}}$. Write $A, A_{\mathbb{C}}, G, K, K_{\mathbb{C}}, N$ and $N_{\mathbb{C}}$ for the analytic subgroups of 
$G_{\mathbb{C}}$ with Lie algebra $\mathfrak{a}, \mathfrak{a}_{\mathbb{C}}, \mathfrak{g}, \mathfrak{k}, \mathfrak{k}_{\mathbb{C}}, \mathfrak{n}$ and $\mathfrak{n}_{\mathbb{C}}$. The Weyl group of $\Sigma$ is given by $\mathcal{W}=N_{K}(\mathfrak{a}) / Z_{K}(\mathfrak{a})$.

For every $\alpha \in \Sigma$ we define a character $\xi_{\alpha}$ of $A_{\mathbb{C}}$ by

$$
\xi_{\alpha}(a)=a^{\alpha}:=e^{\alpha(\log a)} .
$$

Regular and singular elements in $A_{\mathbb{C}}$ are defined by

$$
A_{\mathbb{C}, \text { reg }}=\left\{a \in A_{\mathbb{C}}:(\forall \alpha \in \Sigma) \xi_{\alpha}^{2}(a) \neq 1\right\} \quad \text { and } \quad A_{\mathbb{C}, \text { sing }}=A_{\mathbb{C}} \backslash A_{\mathbb{C}, \text { reg }} .
$$

1.2. Algebraic aspects and categorical quotients. Write $X=G / K$ for the Riemannian symmetric space associated with $G$ and $K$. We denote by

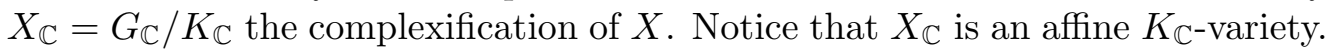

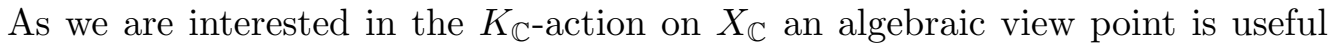
for our geometric studies.

The results collected below are not new, but sometimes we include short proofs for readability. We first recall the basic facts concerning affine varieties with reductive group action.

Let $H$ be a complex reductive group and $V$ be an affine $H$-variety. Define semisimple and regular elements in $V$ by

$$
V_{s}=\{v \in V: H v \text { is closed }\}
$$

and

$$
V_{r}=\{v \in V: \operatorname{dim} H v \text { is maximal }\} .
$$

Further we set $V_{r s}=V_{r} \cap V_{s}$.

Denote by $\mathbb{C}[V]$ the algebra of regular (i.e. polynomial) functions on $V$ and write $\mathbb{C}[V]^{H}$ for the $H$-invariants in $\mathbb{C}[V]$. According to Hilbert, $\mathbb{C}[V]^{H}$ is finitely generated and so corresponds to an affine variety $V / / H$, the categorical quotient of $V$ by $H$. Let us denote by

$$
P: V \rightarrow V / / H
$$

the map corresponding to the inclusion $\mathbb{C}[V]^{H} \hookrightarrow \mathbb{C}[V]$. Recall from $[6$, II.3.2-3] the following properties of $P$.

Lemma 1.2.1. The quotient map $P: V \rightarrow V / / H$ has the following properties:

(1) Every fiber of $P$ contains a unique closed orbit. In particular $P$ is onto.

(2) $\left(\forall v \in V_{s}\right) P^{-1}(P(v))=\{w \in V: v \in \overline{H w}\}$.

(3) $\left(\forall v \in V_{r s}\right) P^{-1}(P(v))=H v$.

Let us now switch to our special situation of $V=X_{\mathbb{C}}$ and $H=K_{\mathbb{C}}$. We denote by $x_{o}=K_{\mathbb{C}}$ the base point in $X_{\mathbb{C}}$. Then, as a special case of general results of Matsuki [10], we obtain that: 
Lemma 1.2.2. The following assertions hold:

(1) $X_{\mathbb{C}, s}=K_{\mathbb{C}} A_{\mathbb{C}} \cdot x_{o}$

(2) $X_{\mathbb{C}, r s}=K_{\mathbb{C}} A_{\mathbb{C}, \mathrm{reg}} \cdot x_{o}$.

We continue with a discussion of the set $X_{\mathbb{C}, r s}$. Define a finite 2-group by $F=A_{\mathbb{C}} \cap K_{\mathbb{C}}$. Notice that $\mathcal{W}$ normalizes $F$ and that both sets $A_{\mathbb{C}, \text { reg }}$ and $A_{\mathbb{C}, \text { sing }}$ are invariant under multiplication by $F$. Write $M_{\mathbb{C}}=Z_{K_{\mathbb{C}}}(A)$. We define a right action of $\mathcal{W}$ on $K_{\mathbb{C}} / M_{\mathbb{C}} \times A_{\mathbb{C}, \mathrm{reg}} / F$ by $\left(k M_{\mathbb{C}}, a F\right) . w=\left(k w M_{\mathbb{C}}, w^{-1} a w F\right)$. The resulting quotient will be denoted by $K_{\mathbb{C}} / M_{\mathbb{C}} \times_{\mathcal{W}} A_{\mathbb{C}, \mathrm{reg}} / F$. Then it was essentially shown in [6, Lemma 2.1] that:

Proposition 1.2.3. The set $X_{\mathbb{C}, r s}$ is open and dense in $X_{\mathbb{C}}$. Moreover, the mapping

$$
\Phi: K_{\mathbb{C}} / M_{\mathbb{C}} \times_{\mathcal{W}} A_{\mathbb{C}, \mathrm{reg}} / F \rightarrow X_{\mathbb{C}, r s}, \quad\left[k M_{\mathbb{C}}, a F\right] \mapsto k a . x_{o}
$$

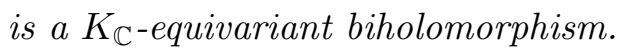

In the remainder of this subsection we discuss more specifically the structure of the quotient mapping $P: X_{\mathbb{C}} \rightarrow X_{\mathbb{C}} / K_{\mathbb{C}}$.

Recall that the algebra $\mathbb{C}\left[X_{\mathbb{C}}\right]^{K_{\mathbb{C}}}$ of invariants is free in $n=\operatorname{dim}_{\mathbb{R}} \mathfrak{a}$ generators, say

$$
\mathbb{C}\left[X_{\mathbb{C}}\right]^{K_{\mathbb{C}}}=\mathbb{C}\left[p_{1}, \ldots, p_{n}\right] \simeq \mathbb{C}\left[x_{1}, \ldots, x_{n}\right] .
$$

Thus $X_{\mathbb{C}} / / K_{\mathbb{C}}=\mathbb{C}^{n}$ and the projection mapping is given by

$$
P: X_{\mathbb{C}} \rightarrow \mathbb{C}^{n}, \quad z \mapsto\left(p_{1}(z), \ldots, p_{n}(z)\right) .
$$

Notice that $A_{\mathbb{C}} / F \rightarrow A_{\mathbb{C}} \cdot x_{o}, a F \mapsto a . x_{o}$ is a biholomorphism. We denote by $P_{A}: A_{\mathbb{C}} \cdot x_{o} \rightarrow \mathbb{C}^{n}$ the restriction of the projection $P$ to $A_{\mathbb{C}} \cdot x_{o}$. We summarize some (known) facts of the map $P_{A}$ :

Proposition 1.2.4. The restriction $P_{A}: A_{\mathbb{C}} \cdot x_{o} \rightarrow \mathbb{C}^{n}$ of $P$ to $A_{\mathbb{C}} \cdot x_{o}$ has the following properties:

(1) The map $P_{A}$ is holomorphic onto open finite and proper.

(2) For every $z \in A_{\mathbb{C}} \cdot x_{o}$ the fiber of $P_{A}$ over $P_{A}(z)=P(z)$ is $\mathcal{W} . z$.

(3) The restriction of $P_{A}$ to the regular elements

$$
\left.P_{A}\right|_{A_{\mathbb{C}, \mathrm{reg}} \cdot x_{o}}: A_{\mathbb{C}, \mathrm{reg}} \cdot x_{o} \rightarrow P\left(A_{\mathbb{C}, \mathrm{reg}} \cdot x_{o}\right)
$$

is a covering map.

Proof. As $P$ is a polynomial map, its restriction $P_{A}$ is holomorphic. Next $P_{A}$ is onto because of Lemma 1.2.1(1) and Lemma 1.2.2(1). We do not show that $P_{A}$ is proper but remark that it is a known fact which can be deduced from the fact that the polynomials $p_{1}, \ldots, p_{n}$ are the elementary spherical functions. 
Standard finite dimensional representation theory then gives the properness of $P_{A}$.

By standard structure theory we have for $z, z^{\prime} \in A_{\mathbb{C}} \cdot x_{o}$ that

$$
K_{\mathbb{C}} z=K_{\mathbb{C}} z^{\prime} \quad \Longleftrightarrow \quad(\exists w \in \mathcal{W}) w \cdot z=z^{\prime}
$$

Thus Lemma 1.2.1(1) and Lemma 1.2.2(1) give us that $P_{A}$ is finite as well as the second assertion 2 . in the proposition.

That $P_{A}$ is open now follows from the properness and 2 . This completes the proof of 1.

Finally, the third assertion 3. follows from 1. and 2.

If $M$ is a complex manifold, then we write $\mathcal{O}(M)$ for the space of holomorphic functions on $M$. If in addition a group $L$ acts on $M$, then we denote by $\mathcal{O}(M)^{L}$ the subspace of $\mathcal{O}(M)$ which consists of $L$-invariant functions. Then we obtain from Proposition 1.2.4 the following (known) corollary:

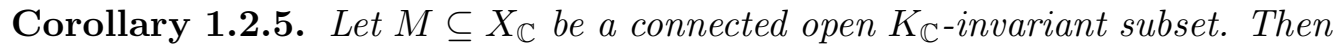
$M_{A}=M \cap A_{\mathbb{C}} \cdot x_{o}$ is open and $\mathcal{W}$-invariant in $A_{\mathbb{C}} \cdot x_{o}$ and the restriction mapping

$$
\operatorname{Res}: \mathcal{O}(M)^{K_{\mathbb{C}}} \rightarrow \mathcal{O}\left(M_{A}\right)^{\mathcal{W}},\left.\quad f \mapsto f\right|_{M_{A}}
$$

is an isomorphism.

Proof. Let us first show that Res is injective. Let $f \in \mathcal{O}(M)^{K_{\mathbb{C}}}$ such that

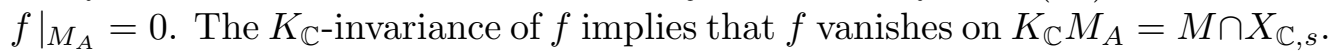
As $X_{\mathbb{C}, s} \cap M$ is dense in $M$ by Proposition 1.2.3, it follows that $f \equiv 0$. Hence Res is injective.

To show that Res is onto, let $F \in \mathcal{O}\left(M_{A}\right)$. We have to show that there exists an $f \in \mathcal{O}(M)^{K_{\mathbb{C}}}$ such that $\left.f\right|_{M_{A}}=F$. Let us define a function on $M$ by

$$
(\forall z \in M) \quad f(z)=F\left(P_{A}^{-1}(P(z))\right) .
$$

This map $f$ is well defined as every fiber of $P_{A}$ is a $\mathcal{W}$-orbit (cf. Proposition 1.2.4(2)) and $F$ is invariant under $\mathcal{W}$. The properness of $P_{A}$ gives us that $f$ is continuous. It is also clear that $\left.f\right|_{M_{A}}=F$ and that $F$ is $K_{\mathbb{C} \text {-invariant. It }}$ remains to see that $f$ is holomorphic. From Proposition 1.2.4(3) we deduce that $f$ is holomorphic on $X_{\mathbb{C}, r s} \cap M$. As $f$ is also continuous, the Riemann removable singularity theorem applies and yields the holomorphicity of $f$.

1.3. Complex crowns. Define a $\mathcal{W}$-invariant, convex and relatively compact subset of $\mathfrak{a}$ by

$$
\Omega=\left\{Y \in \mathfrak{a}:(\forall \alpha \in \Sigma)|\alpha(Y)|<\frac{\pi}{2}\right\} .
$$

Following [1] we define the complex crown $\Xi$ of $X$ by

$$
\Xi=G \exp (i \Omega) \cdot x_{o} \subseteq X_{\mathbb{C}}
$$


It was shown in [1] that $\Xi$ is an open $G$-invariant neighborhood of $X$ with proper $G$-action. Notice that $\Xi$ is independent of the choice of $\mathfrak{a}$ hence canonically attached to $X$. Recently it was shown by many authors that $\Xi$ is actually Stein.

For a subset $\omega \subseteq 2 \Omega$ we define the set

$$
T(\omega)=A \exp (i \omega)
$$

and notice that $T_{\omega}$ is homeomorphic to $\mathfrak{a}+i \omega$ via the exponential mapping $\left.\exp \right|_{\mathfrak{a}_{\mathbb{C}}}: \mathfrak{a}_{\mathbb{C}} \rightarrow A_{\mathbb{C}}$. We recall that $T(4 \Omega) \cap F=\{1\}$. As $F$ is 2-group, it hence follows that the mapping $T(2 \Omega) \rightarrow X_{\mathbb{C}}, a \mapsto a . x_{o}$ is an injection.

Another important feature of the crown is that

$$
\Xi \subseteq N_{\mathbb{C}} T(\Omega) \cdot x_{o}
$$

(cf. [2]). In particular we have a well defined holomorphic mapping

$$
a: \Xi \rightarrow T(\Omega), \quad \text { nu. } x_{o} \mapsto u \quad\left(n \in N_{\mathbb{C}}, u \in T(\Omega)\right)
$$

Further we have the complex convexity theorem [2] which states that

$$
(\forall Y \in \Omega) \quad \operatorname{Im} \log a\left(G \exp (i Y) \cdot x_{o}\right) \subseteq \operatorname{conv}(\mathcal{W} Y)
$$

Here $\operatorname{conv}(\cdot)$ denotes the convex hull of $(\cdot)$.

Set $\mathfrak{a}^{+}=\left\{Y \in \mathfrak{a}:\left(\forall \alpha \in \Sigma^{+}\right) \alpha(Y)>0\right\}$ and $A^{+}=\exp \left(\mathfrak{a}^{+}\right)$. Later we will need also the following subsets of $\Omega$ :

$$
\Omega^{+}=\Omega \cap \mathfrak{a}^{+} \text {and } \Omega^{\prime}=\mathcal{W} \Omega^{+} .
$$

It is useful to introduce some further terminology and define for very $\mathcal{W}$ invariant convex subset $\omega \subseteq 2 \Omega$ the $K_{\mathbb{C} \text {-invariant set }}$

$$
X_{\mathbb{C}, \omega}=K_{\mathbb{C}} T(\omega) \cdot x_{o}
$$

in $X_{\mathbb{C}}$. Notice that even for $\omega$ open the set $X_{\mathbb{C}, \omega}$ is not open in $X_{\mathbb{C}}$. To overcome this difficulty we define

$$
\widehat{X}_{\mathbb{C}, \omega}=P^{-1}\left(P\left(T(\omega) \cdot x_{o}\right)\right)
$$

From the definition it follows immediately that $\widehat{X}_{\mathbb{C}, \omega}$ is a $K_{\mathbb{C} \text {-invariant subset }}$ of $X_{\mathbb{C}}$ containing $X_{\mathbb{C}, \omega}$. Moreover it follows from Proposition 1.2.4(1) that $\widehat{X}_{\mathbb{C}, \omega}$ is open if $\omega$ is open. 


\section{The convexity theorem}

For $Y \in \mathfrak{a}$ let us denote by $\operatorname{co}(Y)$ the convex hull of the Weyl group orbit $\mathcal{W} Y$. We start with stating the main result.

Theorem 2.1. Let $Y \in \Omega$. Then

$$
G \exp (i Y) \cdot x_{o} \bigcap X_{\mathbb{C}, s} \subseteq X_{\mathbb{C}, \operatorname{co}(Y)}=K_{\mathbb{C}} T(\operatorname{co}(Y)) \cdot x_{o}
$$

In particular,

$$
G \exp (i Y) \cdot x_{o} \subseteq \widehat{X}_{\mathbb{C}, \operatorname{co}(Y)}
$$

Before we start proving Theorem 2.1 let us mention an interesting consequence:

Corollary 2.2. The following inclusions hold:

(1) $\Xi \bigcap X_{\mathbb{C}, s} \subseteq X_{\mathbb{C}, \Omega}$.

(2) $\Xi \subseteq \widehat{X}_{\mathbb{C}, \Omega}$.

It seems to us that giving a direct geometric proof of Theorem 2.1 is rather difficult. Instead we will proceed indirectly. We will use spherical functions on $X$. These functions have an analytic continuation to a holomorphic function on $\Xi$ as well as to a $\widehat{X}_{\mathbb{C}, 2 \Omega}$ (cf. [8]). Below we will give pointwise estimates of the analytically extended spherical functions which are uniform in the spectral parameter. From that we will deduce Theorem 2.1.

2.1. Spherical functions. We begin with recalling Harish-Chandra's integral representation of spherical functions. For $\alpha \in \Sigma$ we set $m_{\alpha}=\operatorname{dim} \mathfrak{g}^{\alpha}$ and define $\rho=\frac{1}{2} \sum_{\alpha \in \Sigma^{+}} m_{\alpha} \alpha \in \mathfrak{a}^{*}$.

If $\lambda \in \mathfrak{a}_{\mathbb{C}}^{*}$, then one defines the spherical function on $X$ with parameter $\lambda$ to be the $K$-invariant eigenfunction

$$
\varphi_{\lambda}(g K)=\int_{K} a(k g)^{\rho-\lambda} d k \quad(g \in G) .
$$

Here $d k$ is the normalized Haar measure on the compact group $K$. It follows from (1.3.2) that $\varphi_{\lambda}$ has a holomorphic extension to $\Xi$ which we will denote by $\widetilde{\varphi}_{\lambda}$ (cf. [8]).

On the other hand $\left.\varphi_{\lambda}\right|_{A}$ extends to a $\mathcal{W}$-invariant holomorphic function on $T(2 \Omega)$ [8]. Thus Corollary 1.2.5 applied to $M=\widehat{X}_{\mathbb{C}, 2 \Omega}$ implies:

Proposition 2.1.1. Let $\lambda \in \mathfrak{a}_{\mathbb{C}}^{*}$. Then $\varphi_{\lambda}$ uniquely extends to a $K_{\mathbb{C} \text {-invariant }}$ holomorphic function on $\widehat{X}_{\mathbb{C}, 2 \Omega}$.

We shall prove point-wise estimates for analytically continued spherical functions which are uniform in the spectral paramter. The upper bound is easy to obtain: 
Lemma 2.1.2. Let $Q \subseteq \Xi$ be a compact subset. Then there exists a constant $C>0$ such that for all $g \exp (i Y) \cdot x_{o} \in Q$ with $g \in G, Y \in \Omega$ one has

$$
\left(\forall \lambda \in i \mathfrak{a}^{*}\right) \quad\left|\widetilde{\varphi}_{\lambda}\left(g \exp (i Y) \cdot x_{o}\right)\right| \leq C \cdot \sup _{w \in \mathcal{W}} e^{i \lambda(w Y)} .
$$

Proof. Fix $g \exp (i Y) \cdot x_{o} \in Q$ and recall the definition

$$
\widetilde{\varphi}_{\lambda}\left(g \exp (i Y) \cdot x_{o}\right)=\int_{K} a(k g \exp (i Y))^{\rho-\lambda} d k .
$$

Thus (1.3.3) implies that

$$
\left|\widetilde{\varphi}_{\lambda}\left(g \exp (i Y) \cdot x_{o}\right)\right| \leq \int_{K}\left|a(k g \exp (i Y))^{\rho-\lambda}\right| d k \leq C \cdot \sup _{w \in \mathcal{W}} e^{i \lambda(w Y)}
$$

with

$$
C=\sup _{z \in Q} \int_{K}\left|a(k z)^{\rho}\right| d k<\infty .
$$

Now we switch to the lower estimates. For that we will use Harish-Chandra's power series expansion of spherical function (cf. [4]).

We briefly recall the standard notation. For $\sigma \in \mathfrak{a}_{\mathbb{C}}^{*}$ and $a \in T(\Omega)$ we write $a^{\sigma}=e^{\sigma(\log a)}$. Let us call an element $\lambda \in \mathfrak{a}_{\mathbb{C}}^{*}$ regular if $\langle\lambda, \alpha\rangle \neq 0$ for all $\alpha \in \Sigma$. Define a semigroup $\Lambda=\mathbb{N}_{0}\left[\Sigma^{+}\right] \subseteq \mathfrak{a}^{*}$. As usual we denote by $\mathbf{c}(\lambda)$ the HarishChandra c-function. This is a meromorphic function on $\mathfrak{a}_{\mathbb{C}}^{*}$ with no poles on the regular elements in $i \mathfrak{a}^{*}$; it can be explicitely computed (Gindikin-Karpelevic formula, see [4, Ch. IV, Th. 6.14]).

Fix $\lambda \in i \mathfrak{a}^{*}$ regular. Then for $a \in A^{+}$Harish Chandra's expansion of $\varphi_{\lambda}$ is given as follows (cf. [4, Ch. IV, Th. 5.5]):

$$
\varphi_{\lambda}(a)=\sum_{w \in \mathcal{W}} \mathbf{c}(w \lambda) \cdot a^{w \lambda-\rho} \cdot \Psi_{w \lambda}(a)
$$

where

$$
\Psi_{w \lambda}(a)=\sum_{\mu \in \Lambda} \Gamma_{\mu}(w \lambda) a^{-\mu}
$$

For $\sigma \in i \mathfrak{a}^{*}$ the coefficients $\Gamma_{\mu}(\sigma)$ are defined recursively by $\Gamma_{0}(\sigma)=1$ and

$$
\Gamma_{\mu}(\sigma)=\frac{2}{\langle\mu, \mu\rangle+2\langle\mu, \sigma\rangle} \sum_{\alpha \in \Sigma^{+}} m_{\alpha} \sum_{k \geq 1} \Gamma_{\mu-2 k \alpha}(\sigma)(\langle\mu+\rho-2 k \alpha, \alpha\rangle+\langle\alpha, \sigma\rangle) .
$$

The power series $\Psi_{w \lambda}$ are locally abolutely convergent on $A^{+}$. One can deduce this from the following fact ([4, Ch. IV, Lemma 5.6]): for all $H \in \mathfrak{a}^{+}$there is a constant $C_{H}>0$ such that

$$
(\forall \mu \in \Lambda)\left(\forall \lambda \in i \mathfrak{a}^{*}\right) \quad\left|\Gamma_{\mu}(\lambda)\right| \leq C_{H} \cdot e^{\mu(H)} .
$$

It is clear that (2.1.1) is also valid for all $a \in A^{+} \exp (i \Omega)$. 
Lemma 2.1.3. Fix $\lambda_{0} \in i \mathfrak{a}^{*}$. Then the following assertions hold:

(1) For all $\mu \in \Lambda$ the limit

$$
\Gamma_{\mu}\left[\lambda_{0}\right]:=\lim _{t \rightarrow \infty} \Gamma_{\mu}\left(t \lambda_{0}\right)
$$

exists.

(2) The power series

$$
\Psi_{\left[\lambda_{0}\right]}(a)=\sum_{\mu \in \Lambda} \Gamma_{\mu}\left(\left[\lambda_{0}\right]\right) a^{-\mu} \quad\left(a \in A^{+} \exp (i \Omega)\right)
$$

is locally absolutely convergent on $A^{+} \exp (i \Omega)$. In particular, $\Psi_{\left[\lambda_{0}\right]}$ defines a non-zero holomorphic function on $A^{+} \exp (i \Omega)$. Moreover

$$
\Psi_{t \lambda_{0}} \rightarrow \Psi_{\left[\lambda_{0}\right]}
$$

locally uniformly on $A^{+} \exp (i \Omega)$ for $t \rightarrow \infty$.

Proof. 1. Let $\alpha_{1}, \ldots, \alpha_{n}$ be a basis of $\Sigma^{+}$. If $\mu=m_{1} \alpha_{1}+\ldots+m_{n} \alpha_{n} \in \Lambda$, then define its degree by $\operatorname{deg} \mu=m_{1}+\ldots+m_{n}$. We use the recurrence relation (2.1.3) and induction on $\operatorname{deg} \mu$ to deduce that for every $\mu \in \Lambda$ the function

$$
F_{\mu}: \mathbb{R} \rightarrow \mathbb{C}, \quad t \mapsto \Gamma_{\mu}\left(t \lambda_{0}\right)
$$

is rational with no poles. Moreover (2.1.3) implies that $F_{\mu}$ is bounded. Thus if $F_{\mu}=\frac{p_{\mu}}{q_{\mu}}$ with polynomials $p_{\mu}$ and $q_{\mu}$ we obtain that $\operatorname{deg} p_{\mu} \leq \operatorname{deg} q_{\mu}$. From that the assertion follows.

2. This follows from 1. and the estimate (2.1.3).

Proposition 2.1.4. Fix $\lambda_{0} \in i \mathfrak{a}^{*}$ regular. Let $H \in \mathfrak{a}^{+}$and $Y \in \Omega^{\prime}$ and set $a=\exp (H+i Y)$. Then there exists a constant $C_{a, \lambda_{0}} \geq 0$ such that for all $t>>0$ one has

$$
\left|\widetilde{\varphi}_{t \lambda_{0}}\left(a . x_{o}\right)\right| \geq \frac{C_{a, \lambda_{0}}}{t^{p}} \cdot \sup _{w \in \mathcal{W}} e^{i t \lambda_{0}(w Y)}
$$

where $p=\frac{1}{2} \operatorname{dim} N$. Moreover $C_{a, \lambda_{0}}>0$ for all elements $a$ in a dense open subset of $A^{+} \exp \left(i \Omega^{\prime}\right)$.

Proof. A basic result of Harish-Chandra asserts that $\varphi_{\lambda}=\varphi_{w \lambda}$ for all $\lambda \in \mathfrak{a}_{\mathbb{C}}^{*}$ and $w \in \mathcal{W}$. Thus by the regularity of $\lambda_{0}$ and $Y$ we may assume that $\lambda_{0}(i Y)>$ $\lambda_{0}(i w Y)$ for all $w \in \mathcal{W}, w \neq \mathbf{1}$. Therefore it suffices to show that

$$
(\forall t>>0) \quad\left|\widetilde{\varphi}_{t \lambda_{0}}\left(a \cdot x_{o}\right)\right| \geq \frac{C_{a, \lambda_{0}}}{t^{p}} \cdot e^{i t \lambda_{0}(Y)}
$$

for a constant $C_{a, \lambda_{0}} \geq 0$ which is actually positive for $a$ ranging in a dense open subset of $A^{+} \exp \left(i \Omega^{\prime}\right)$. 
From (2.1.1) we obtain that:

$$
\widetilde{\varphi}_{t \lambda_{0}}\left(a . x_{o}\right)=a^{\rho} \cdot \sum_{w \in \mathcal{W}} \mathbf{c}\left(t w \lambda_{0}\right) \cdot e^{i t \lambda_{0}(w Y)} \cdot e^{t \lambda_{0}(w H)} \cdot \Psi_{t w \lambda_{0}}(a)
$$

Contemplating on (2.1.5) we notice the following facts:

- $\left|e^{t \lambda_{0}(w H)}\right|=1$ for all $t \in \mathbb{R}$ and $w \in \mathcal{W}$ as $\lambda_{0}$ is imaginary.

- $\left|a^{\rho}\right|>0$ is independent of $\lambda$.

- $\left|\mathbf{c}\left(t w \lambda_{0}\right)\right|=\left|\mathbf{c}\left(t \lambda_{0}\right)\right|$ for all $w \in \mathcal{W}$ (Maaß-Selberg relations, cf. [4, p. 451, (16)]).

- There is a constant $C>0$ such that $\left|\mathbf{c}\left(t \lambda_{0}\right)\right| \geq \frac{C}{t^{p}}$ for $t>>0$ (cf. [4, Ch. IV, Prop. 7.2]).

- $\Psi_{t w \lambda_{0}}(a)$ is uniformly bounded in $t$ for all $w \in \mathcal{W}$ by Lemma 2.1.2 (2).

Combining these facts with (2.1.5) we obtain (2.1.4) for some $C_{a, \lambda_{0}} \geq 0$. To be more precise we have $C_{a, \lambda_{0}}>0$ provided that $\Psi_{\left[\lambda_{0}\right]}(a) \neq 0$ (cf. Lemma 2.1.3(2)), and this finishes the proof.

2.2. Proof of Theorem 2.1. During the proof we will frequently use the fact that the mapping

$$
G / M \times \Omega^{+} \rightarrow \Xi, \quad(g M, Y) \mapsto g \exp (i Y) \cdot x_{o}
$$

is a diffeomorphism with open and dense image (cf. [9, Cor. 4.2]).

Due to the statement of the theorem we may assume that $Y \in \Omega^{+}$. We now proceed by contradiction. If the statement were false, there would exist a $g \in G$ such that $g \exp (i Y) \cdot x_{o} \in X_{\mathbb{C}, s}$ but $g \exp (i Y) \cdot x_{o} \notin K_{\mathbb{C}} T(\operatorname{co}(Y)) \cdot x_{o}$. Let $g(t), t \in[0,1]$, be a continuous path in $G$ such that $g(0)=1$ and $g(1)=g$. Recall the quotient mapping $P: X_{\mathbb{C}} \rightarrow \mathbb{C}^{n}$ from Subsection 1.2. Define a continuous path in $\mathbb{C}^{n}$ by $\gamma(t)=P\left(g(t) \exp (i Y) \cdot x_{o}\right)$. Then $\gamma(0)=P\left(\exp (i Y) \cdot x_{o}\right) \in$ $P\left(T(\operatorname{co}(Y)) \cdot x_{o}\right)$. As $P_{A}=\left.P\right|_{A_{\mathbb{C}} \cdot x_{o}}$ is proper (cf. Proposition 1.2.4(1)), we conclude that $P\left(T(\operatorname{co}(Y)) \cdot x_{o}\right)$ is closed. Thus there exists a $0 \leq t_{0}<1$ which is maximal with respect to the property that $\gamma(t) \in P\left(T(\operatorname{co}(Y)) \cdot x_{o}\right)$ for all $0 \leq t \leq t_{0}$. Again it follows from Proposition 1.2.4(1) that $P\left(T(\Omega) \cdot x_{o}\right)$ is an open neighborhood of $\gamma\left(t_{0}\right)$. Thus we find a $t_{0}<t_{1} \leq 1$ such that $\gamma(t) \in P\left(T(\Omega) . x_{o}\right)$ for all $0 \leq t \leq t_{1}$. Now pick $t_{0}<t_{2} \leq t_{1}$ such that $\gamma\left(t_{2}\right)=P\left(a . x_{o}\right)$ for some $a \in T(\Omega) \backslash T(\operatorname{co}(Y))$. It is no loss of generality to assume now that $g=g\left(t_{2}\right)$ and reparametrizing $g(t)$ we may also assume that $t_{2}=1$.

To sum up, if the statement were false we find a $g \in G$ and a continuous path $g(t), t \in[0,1]$, in $G$ with $g(0)=\mathbf{1}$ and $g(1)=g$ such that $g(t) \exp (i Y) . x_{o} \in \widehat{X}_{\mathbb{C}, \Omega}$ for all $t \in[0,1]$ and $P\left(g \exp (i Y) \cdot x_{o}\right)=P\left(a . x_{o}\right)$ for some $a \in T(\Omega) \backslash T(\operatorname{co}(Y))$. In particular, it follows from Proposition 2.1.1 that

$$
\left(\forall \lambda \in \mathfrak{a}_{\mathbb{C}}^{*}\right) \quad \widetilde{\varphi}_{\lambda}\left(g \exp (i Y) \cdot x_{o}\right)=\widetilde{\varphi}_{\lambda}\left(a \cdot x_{o}\right)
$$


Using (2.2.1) we can vary $g$ and $Y$ slighty to obtain $a \in A^{+} \exp \left(i \Omega^{\prime}\right)$, say $a=\exp (H+i Z)$ for some $H \in \mathfrak{a}^{+}$and $Z \in \Omega^{\prime}$.

As $Z \in \Omega \backslash \operatorname{co}(Y)$, we can use the theorem of Hahn-Banach to find a $\lambda_{0} \in i \mathfrak{a}^{*}$ regular such that $\left|\lambda_{0}(Z)\right|>\left|\lambda_{0}(w Y)\right|$ for all $w \in \mathcal{W}$.

Lemma 2.1.2 implies

$$
(\forall t>0) \quad\left|\widetilde{\varphi}_{t \lambda_{0}}\left(g \exp (i Y) \cdot x_{o}\right)\right| \leq C \cdot \sup _{w \in \mathcal{W}} e^{t i \lambda_{0}(w Y)}
$$

for a constant $C>0$.

On the other hand (2.2.2) combined with Proposition 2.1.4 gives us for all $t>>0$ that

$$
\left|\widetilde{\varphi}_{t \lambda_{0}}\left(g \exp (i Y) \cdot x_{o}\right)\right|=\left|\widetilde{\varphi}_{t \lambda_{0}}\left(\exp (H+i Z) \cdot x_{o}\right)\right| \geq \frac{C^{\prime}}{t^{p}} \cdot \sup _{w \in W} e^{t i \lambda_{0}(w Z)}
$$

for a constant $C^{\prime} \geq 0$. Moreover varying $g$ and $Y$ slightly if necessary we may assume that $C^{\prime}>0$ (cf. Proposition 2.1.4). Then (2.2.4) clearly contradicts (2.2.3), concluding the proof of the theorem.

2.3. Applications. Define a subset of $\mathfrak{a}_{\mathbb{C}}^{*}$ by

$$
\Pi=\left\{\lambda \in \mathfrak{a}_{\mathbb{C}}^{*}: \varphi_{\lambda} \text { is positive definite }\right\}
$$

Recall that $i \mathfrak{a}^{*} \subseteq \Pi$ and that for all $\lambda \in \Pi$ the spherical function $\varphi_{\lambda}$ is bounded on $X$.

Using Theorem 2.1 and the same reasoning as in [7] we now obtain that:

Theorem 2.3.1. Let $\lambda \in \Pi$. Then the analytically continued spherical function $\widetilde{\varphi}_{\lambda}$ is bounded on $\Xi$.

Proof. For $\lambda \in \Pi$ it was established in [8] that $\widetilde{\varphi}_{\lambda}$ is bounded on $X_{\mathbb{C}, \Omega}$. Now the assertion follows from Corollary 2.2.

Another application of Theorem 2.1 is related to the heat kernel which was suggested to us by M. Stenzel. In the sequel $t>0$ denotes a positive number. We denote by $k_{t}(x), x \in X$, the heat kernel on $X$.

Using the spectral resolution of $k_{t}$ it was shown in [9] that $k_{t}$ has an analytic continuation to a holomorphic function on $\Xi$ which we will denote by $\widetilde{k}_{t}$. Then it follows readily from Theorem 2.1, Lemma 2.1.2 and the spectral resolution of $k_{t}$ that:

Theorem 2.3.2. Fix $t>0$ and let $k_{t}$ be the heat kernel on $X$. Then the analytically continued heat kernel $\widetilde{k}_{t}$ is bounded on $\Xi$. 


\section{Examples: Symmetric matrices}

In this section we will illustrate Theorem 2.1 for the choices of $G=\operatorname{Sl}(n, \mathbb{R})$ and $G=\operatorname{Sl}(n, \mathbb{C})$. Then $X=G / K$ has a canonical realization in real/complex symmetric matrices. It might be interesting to observe that even in these classical cases Theorem 2.1 seems to be new and likely forfeits a proof using only techniques from elementary linear algebra.

3.1. Real symmetric matrices. Let now $G=\mathrm{Sl}(n, \mathbb{R})$ and $K=\mathrm{SO}(n)$. Then $G_{\mathbb{C}}=\operatorname{Sl}(n, \mathbb{C})$ and $K_{\mathbb{C}}=\operatorname{SO}(n, \mathbb{C})$. The mapping $g K_{\mathbb{C}} \mapsto g g^{t}$ identifies $X_{\mathbb{C}}$ with the complex symmetric matrices of determinant one

$$
X_{\mathbb{C}}=\left\{Z \in M(n, \mathbb{C}): Z=Z^{t}, \operatorname{det} Z=1\right\} .
$$

Within this identification $X$ is the set of all real positive definite symmetric matrices of determinant one

$$
X=\left\{Y \in M(n, \mathbb{R}): Y=Y^{t}, Y>>0, \operatorname{det} Y=1\right\} .
$$

We choose $A$ to be the positive diagonal matrices in $G=\operatorname{Sl}(n, \mathbb{R})$. Then $\mathcal{W}$ becomes the symmetric group $\mathfrak{S}_{n}$ in $n$ letters permuting the diagonal entries. The set $\Omega$ is given by

$$
\Omega=\left\{\operatorname{diag}\left(y_{1}, \ldots, y_{n}\right) \in M(n, \mathbb{R}): \sum_{i=1}^{n} y_{i}=0,\left|y_{i}-y_{j}\right|<\frac{\pi}{2}\right\}
$$

Accordingly we have

$$
T(\Omega)=\left\{a=\operatorname{diag}\left(a_{1}, \ldots, a_{n}\right) \in \operatorname{Sl}(n, \mathbb{C}):(\forall 1 \leq i, j \leq n) \quad \operatorname{Re}\left(\frac{a_{i}}{a_{j}}\right)>0\right\}
$$

Let us write $D_{\mathbb{R}}$ for the diagonal matrices in $X$ and $D_{\mathbb{C}}$ for the diagonal matrices in $X_{\mathbb{C}}$. Notice that the mapping $A_{\mathbb{C}} \rightarrow D_{\mathbb{C}}, a \mapsto a . x_{o}=a^{2}$ is the square mapping. If $D(\Omega)$ denotes the isomorphic image of $T(\Omega)$ in $X_{\mathbb{C}}$, then we have

$$
D(\Omega)=\left\{a=\operatorname{diag}\left(a_{1}, \ldots, a_{n}\right) \in X_{\mathbb{C}}:(\forall 1 \leq i, j \leq n) \quad \frac{a_{i}}{a_{j}} \in \mathbb{C}_{+}\right\},
$$

where $\left.\left.\mathbb{C}_{+}=\mathbb{C} \backslash\right]-\infty, 0\right]$.

Notice that

$$
\Xi=\bigcup_{g \in G} g D(\Omega) g^{t}
$$

Now Theorem 2.1 in this special situation reads as follows: 
Proposition 3.1.1. Let $Y=\operatorname{diag}\left(y_{1}, \ldots, y_{n}\right)$ be a real diagonal matrix with zero trace and $\left|y_{i}-y_{j}\right|<\pi$ for all $1 \leq i, j \leq n$. Then

$$
\operatorname{co}(Y)=\operatorname{conv}\left\{\operatorname{diag}\left(y_{\sigma(1)}, \ldots, y_{\sigma(n)}\right): \sigma \in \mathfrak{S}_{n}\right\}
$$

and

$$
\left[\bigcup_{g \in \operatorname{Sl}(n, \mathbb{R})} g e^{i Y} g^{t}\right] \bigcap\left[\bigcup_{k \in \operatorname{SO}(n, \mathbb{C})} k D_{\mathbb{C}} k^{-1}\right] \subseteq \bigcup_{k \in \operatorname{SO}(n, \mathbb{C})} k D_{\mathbb{R}} e^{i \operatorname{co}(Y)} k^{-1} .
$$

3.2. Complex symmetric matrices. Let us now consider the case of $G=$ $\mathrm{Sl}(n, \mathbb{C})$ and $K=\mathrm{SU}(n)$. Then we have canonical biholomorphisms $K_{\mathbb{C}} \simeq G$ and $X_{\mathbb{C}} \simeq G$. Within this identifications the actions of $G$ and $K_{\mathbb{C}}$ on $X_{\mathbb{C}}$ are given by

$$
\begin{aligned}
G \times X_{\mathbb{C}} & \rightarrow X_{\mathbb{C}}, \quad(g, z) \mapsto g z g^{*}, \\
K_{\mathbb{C}} \times X_{\mathbb{C}} & \rightarrow X_{\mathbb{C}}, \quad(k, z) \mapsto k z k^{-1}
\end{aligned}
$$

(cf. [3, Sect. 3]). As before we choose $A<G$ to be the group of diagonal matrices with positive entries and write $D(\Omega)$ for the faithful image of $T(\Omega)$ in $X_{\mathbb{C}}=G$. Then

$$
D(\Omega)=\left\{a=\operatorname{diag}\left(a_{1}, \ldots, a_{n}\right) \in X_{\mathbb{C}}:(\forall 1 \leq i, j \leq n) \quad \frac{a_{i}}{a_{j}} \in \mathbb{C}_{+}\right\} .
$$

In particular we obtain

$$
\Xi=\bigcup_{g \in \operatorname{Sl}(n, \mathbb{C})} g D(\Omega) g^{*} .
$$

With the notation introduced above Theorem 2.1 now reads as follows:

Proposition 3.2.1. Let $Y=\operatorname{diag}\left(y_{1}, \ldots, y_{n}\right)$ be a real diagonal matrix with zero trace and $\left|y_{i}-y_{j}\right|<\pi$ for all $1 \leq i, j \leq n$. Then

$$
\left[\bigcup_{g \in \operatorname{Sl}(n, \mathbb{C})} g e^{i Y} g^{*}\right] \bigcap\left[\bigcup_{k \in \operatorname{Sl}(n, \mathbb{C})} k D_{\mathbb{C}} k^{-1}\right] \subseteq \bigcup_{k \in \operatorname{Sl}(n, \mathbb{C})} k D_{\mathbb{R}} e^{i \operatorname{co}(Y)} k^{-1}
$$

where $\operatorname{co}(Y)=\operatorname{conv}\left\{\operatorname{diag}\left(y_{\sigma(1)}, \ldots, y_{\sigma(n)}\right): \sigma \in \mathfrak{S}_{n}\right\}$.

\section{Acknowledgments}

The author would like to thank M. Otto, C. Rader and M. Stenzel for several useful discussions. 


\section{References}

[1] D. Akhiezer, and S. Gindikin, On Stein extensions of real symmetric spaces, Math. Ann. 286 (1990), 1-12.

[2] S. Gindikin, and B. Krötz, Invariant Stein Domains in Stein Symmetric Spaces and a Nonlinear Complex Convexity Theorem, IMRN 18 (2002), 959-971.

[3] - Complex crowns of Riemannian symmetric spaces and non-compactly causal symmetric spaces, Trans. Amer. Math. Soc. 354 (2002), 3299-3327.

[4] S. Helgason, Groups and Geometric Analysis, Academic Press, 1984.

[5] B. Kostant, On convexity, the Weyl group and the Iwasawa decomposition, Ann. Sci. Ecole Norm. Sup. 6 (1973), 413-455.

[6] H. Kraft, Geometrische Methoden in der Invariantentheorie, Vieweg, 1984.

[7] B. Krötz, and M. Otto, A convexity property for the $\mathrm{SO}(2, \mathbb{C})$ double coset decomposition of $\mathrm{Sl}(2, \mathbb{C})$ and applications to spherical functions, Math. Z., to appear.

[8] B. Krötz, and R. Stanton, Holomorphic extension of representations: (I) automorphic functions, Ann. Math., to appear.

[9] , Holomorphic extension of representations: (II) geometry and harmonic analysis, preprint.

[10] T. Matsuki, Double coset decompositions of reductive Lie groups arising from two involutions, J. Algebra 197 (1997), 49-91.

[11] K.-H. Neeb, A general non-linear convexity theorem, Forum Math. 9 (1997), 613-640.

[12] V.S. Varadarajan, The Method of Stationary Phase and Applications to Geometry and Analysis on Lie Groups, Algebraic and Analytic Methods in Representation Theory, Associated Press, 1997.

Department of Mathematics, 1222 University of Oregon, Eugene OR 97403-1222

E-mail address: kroetz@math.uoregon.edu 